\title{
FUNCTIONAL OUTCOME OF OPEN PHALANGEAL AND METACARPAL FRACTURES TREATED WITH EXTERNAL FIXATION
}

Kishore Kannan'1, Mathivanan Palaniappan², Srinivasan Anbu³, Kalyanasundaram Kolundan', Ramesh Prabu Ganesan ${ }^{5}$, Subramanian Karunanithi 6

\begin{abstract}
${ }^{1}$ Assistant Professor, Department of Orthopaedics, KAPV Government Medical College/M.G.M Govt. Hospital, Tiruchirapalli. 2 Professor, Department of Orthopaedics, KAPV Government Medical College/M.G.M Govt. Hospital, Tiruchirapalli. ${ }_{3}^{3}$ Associate Professor, Department of Orthopaedics, KAPV Government Medical College/M.G.M Govt. Hospital, Tiruchirapalli. ${ }^{4}$ Associate Professor, Department of Orthopaedics, KAPV Government Medical College/M.G.M Govt. Hospital, Tiruchirapalli. ${ }^{5}$ Assistant Professor, Department of Orthopaedics, KAPV Government Medical College/M.G.M Govt. Hospital, Tiruchirapalli. ${ }^{6}$ Assistant Professor, Department of Orthopaedics, KAPV Government Medical College/M.G.M Govt. Hospital, Tiruchirapalli.
\end{abstract}

\section{ABSTRACT}

\section{BACKGROUND}

This is a prospective study conducted from 2008 to 2016 in Department of Orthopaedics, K.A.P.V Govt. Medical College/M.G.M Govt. Hospital, Tiruchirapalli. We treated 69 patients with 63 Phalangeal and 15 Metacarpal fractures with open or accompanied by soft tissue injuries with mini external fixation device.

\section{MATERIALS AND METHODS}

The common age group affected was 21-30 years with mean age being 35.5 years (13-60 years). Most of the injuries were caused by industrial accidents (57.6\%). In 34.6\% of cases, the dominant hand was involved. All patients were treated with wound debridement, external fixation and early mobilisation of the fingers. The follow-up period ranged between $2.3-8.2$ years (mean 4.4 years). Complications occurred in ten fractures. Loosening of the pin (6) was the most common complication. Except two, all the fractures healed.

\section{RESULTS}

The functional recovery was analysed using the American Society for Surgery of the Hand (ASSH) criteria of TAM. Results were good or excellent in $86.7 \%$ of metacarpal fractures and in $85.7 \%$ of Phalangeal fractures with overall result of about $85.9 \%$. Poor results occurred in about $14.1 \%$ fractures. Majority of these cases had multiple fractures or accompanied with severe soft tissue injuries.

\section{CONCLUSION}

To conclude external fixation is an adequate alternative treatment for unstable open Phalangeal and metacarpal fractures, which are accompanied by severe soft tissue injuries.

\section{KEYWORDS}

Open Phalanx Fractures, Open Metacarpal Fractures, External Fixation.

HOW TO CITE THIS ARTICLE: Kannan K, Palaniappan M, Anbu S, et al. Functional outcome of open phalangeal and metacarpal fractures treated with external fixation. J. Evolution Med. Dent. Sci. 2016;5(77):5716-5720, DOI: 10.14260/jemds/2016/1289

\section{INTRODUCTION}

Phalangeal and Metacarpal fractures are commonly seen injuries in Emergency Department. In general, these injuries comprise of $17.5 \%$ of all fractures in the body. The problem of fractures of the hand is one of the most fascinating and challenging aspects in hand surgery. Fascinating because of the multiplicity and diversity of these fractures and challenging because of its crippling effects on the delicate gliding system of the tendons. Improper and inadequate treatment may lead to permanent disability. ${ }^{1}$

Most of these fractures can be treated conservatively, but in a relatively small number of patients with unstable or open

Financial or Other, Competing Interest: None.

Submission 22-07-2016, Peer Review 12-09-2016,

Acceptance 19-09-2016, Published 24-09-2016.

Corresponding Author:

Dr. Kishore Kannan,

K-101, Lasan Livings,

C-55, $10^{\text {th }}$ Cross West,

Thillai Nagar, Tiruchirapalli-620018.

E-mail: skdortho@yahoo.co.in

DOI: $10.14260 /$ jemds $/ 2016 / 1289$ fractures, operative treatment is indicated. Conventional methods of internal fixation using ' $\mathrm{K}$ ' wires or mini plates and screws may not be suitable for open fractures accompanied by severe soft tissue injuries. ${ }^{2}$ The use of an external device reduces further damage to the delicate soft tissues and bone, allow better wound care and enable early mobilisation of joints. ${ }^{3}$

The functional aim of hand surgery must not be merely wound healing. The patient should ideally be returned to their pre-injury lifestyle encompassing all vocational domestic and recreational activities without any physical and psychological impairment. However, the ultimate objective of fracture management is to obtain a pain free, stable and mobile hand.

Our prospective study analysed the functional results in a group of patients with open metacarpal and phalangeal fractures with soft tissue injuries treated by external fixation.

\section{ANATOMY OF THE HAND}

The skeleton of the hand and wrist is composed of 19 tubular long bones and 8 carpal bones. These 27 structures are arranged into five rays. Each ray has its base at the 
carpometacarpal articulation. The rays then forms a polyarticulated chains of metacarpal and either two or three phalanges.

The static base or fixed unit of the hand is formed by the second and third metacarpals at their articulation with the transverse carpal arch. This fixed unit is joined on either side by metacarpal of the thumb, ring and little fingers that add mobility to this fixed unit and rotate in response to musculotendinous movements to provide a transverse metacarpal arch essential for grasp and the adaptive movement of the hand.

\section{INCIDENCE}

Fractures of the metacarpals and phalanges are one of the most common fractures of the upper extremity. In general, these fractures comprise $17.5 \%$ of all fractures throughout the body. The outer rays of the hand (Thumb and fifth finger) are more frequently injured. Phalangeal fractures are more common than metacarpal fractures.

- Male-to-female ratio ranges from 1.8:1 to 5.4:1.

- The incidence of metacarpal and phalangeal fracture peaks between 10 and 40 years. Single fracture occurs in $98.6 \%$ of the cases and multiple fractures seen in only $1.4 \%$ of cases. The commonest mode of injury is industrial accident. Others are road traffic accidents, domestic accidents and assaults.

- Unfortunately, metacarpal and phalangeal fractures are often rejected and regarded as trivial injuries.

- Swanson had rightly said - hand fracture can be complicated by deformity from no treatment: stiffness from over treatment and both deformity and stiffness from poor treatment. ${ }^{4}$

\section{Principles of External Fixation}

To be safe and effective an applied fixator should have a low rate of serious complication, non-obstructive and stiff enough to maintain alignment under adverse loading situation. It should facilitate early mobilisation and be adaptable to a wide variety of injury and patient conditions.

Behrens and Searls (1986) found four basic principles, which demand that the applied frame optimally accommodate the vital limb anatomy, injury site access for debridement and secondary procedures, mechanical demands of the patient and injuries and patient comfort. ${ }^{5}$

The following basic characteristics have shown to increase the stiffness of an applied frame and to diminish motion at the fracture site.

- Use static frame for all, except intra- and juxta-articular fractures.

- Two wires must be passed in each fragment.

- If the fracture is too close to the articular surface such that it precludes the use of the two wires in each fragment, the frame must span the joint. At least one wire must be passed in the bone across the joint.

- Pre-stressing of wires must be performed wherever safe.

- Intramedullary wires enhance stability. They may be used when the wires do not penetrate joint surface dangerously and in the absence of contamination/infection.

- Reducing the distance between the two K wires, which ideally must be $6-7 \mathrm{~mm}$.

\section{AIM OF THE STUDY}

It is to study the role of mini external fixation device in the management of metacarpal and phalangeal fractures, which are open or accompanied with soft tissue injuries.

\section{MATERIAL AND METHODS}

In the last eight years, we have operated on 69 patients (60 men and 9 women) with 78 fractures in phalanges and metacarpals by external fixation. The common age group affected was 21-30 yrs. with mean age being 35.5 years (1360 years). Most of the injuries were caused by industrial accidents (57.6\%) and next common is road traffic accident. In $34.6 \%$ of the cases, the dominant hand was involved.

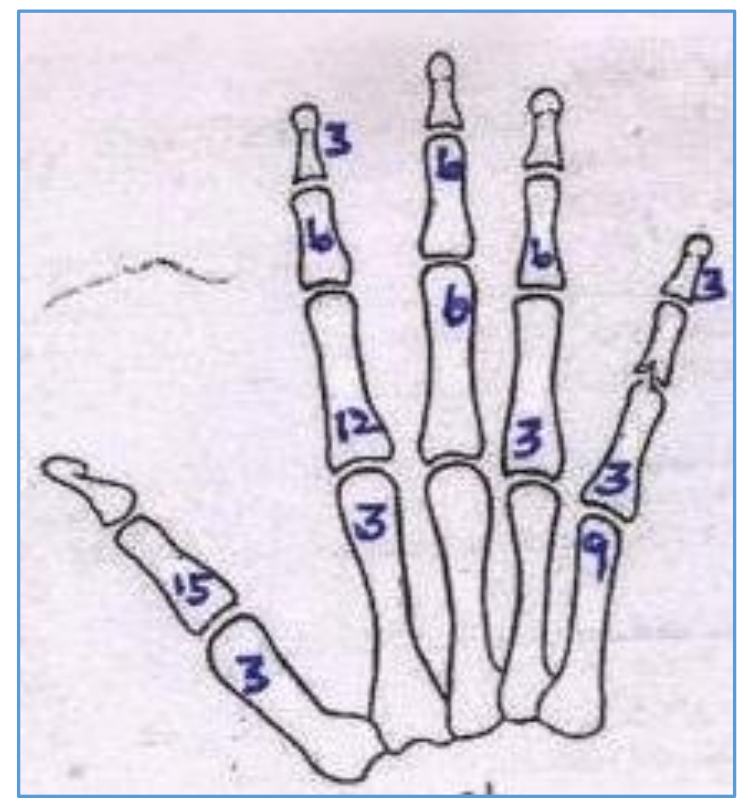

Fig. 1: Distribution of Fractures

The distribution of 63 Phalangeal and 15 Metacarpal fractures are as shown in Figure. 1. The proximal phalanx of the thumb (15 cases) was more often involved than the other bones in the fingers. Fifth metacarpal (9 cases) was found to be involved more commonly than other metacarpals. There were several types of fractures; 30 were comminuted, 24 were transverse, 9 oblique and 15 intraarticular. There were 21 open fractures with severe soft tissue injuries, which needed flap cover and 9 cases were associated with tendon injuries.

As soon as the patient is received in emergency department initial assessment of injury was done, the wound was thoroughly washed with saline and sterile dressing applied and hand elevated. X-ray of the affected hand in two views; anteroposterior and oblique views were taken. Majority of the patients had early operation within 12 hours of injury.

\section{Steps in the Management}

- Wound debridement.

- Reduction.

- Maintenance of reduction with external fixation.

- Repair of deeper structures.

- Timing of wound closure or coverage.

- Post-operative care.

- Hand rehabilitation. 
All the patients except two were operated under regional anaesthesia (axillary, wrist or digital blocks). After thorough wound debridement fractures were reduced under vision and stabilised with external fixator frames. We used static frames ${ }^{6}$ with two parallel $\mathrm{K}$ wires passed in each fragment. In juxta-articular fractures, the frame spanned the joint. Maintaining reduction, the connecting rod was fixed parallel to the lateral border of the finger with clamps.

We used unilateral frame for 63 patients, Ray frame for 9 patients with intraarticular or juxta articular fractures and bilateral frame for 6 patients with intraarticular fractures of the thumb. Lateral surface of the digit presents the safest area for passage of $\mathrm{K}$ wires. Lateral frames are only applicable to the border digits (index and little finger). In case of fractures of middle or ring finger, the frames are oriented in a dorsolateral plane. Postoperative care involved elevation and immediate mobilisation of the adjacent joints of injured digit and uninjured fingers. Plaster support was indicated only if the fracture is associated with severe soft tissue injuries, multiple fractures or associated tendon injuries. Radiographs were taken immediately after surgery and at one to two weeks and at four weeks to check the position and healing.

Patients were discharged when the soft tissue condition permitted. Because of the well-recognised delay in appearance of radio opaque callus, fixator was removed when union was clinically than radiologically evident. The fixators were removed at 3 to 4 weeks. The hand fractures become stable enough by this time to tolerate gentle active range of motion with further remodelling by 8-10 weeks and consolidation by 12 weeks.
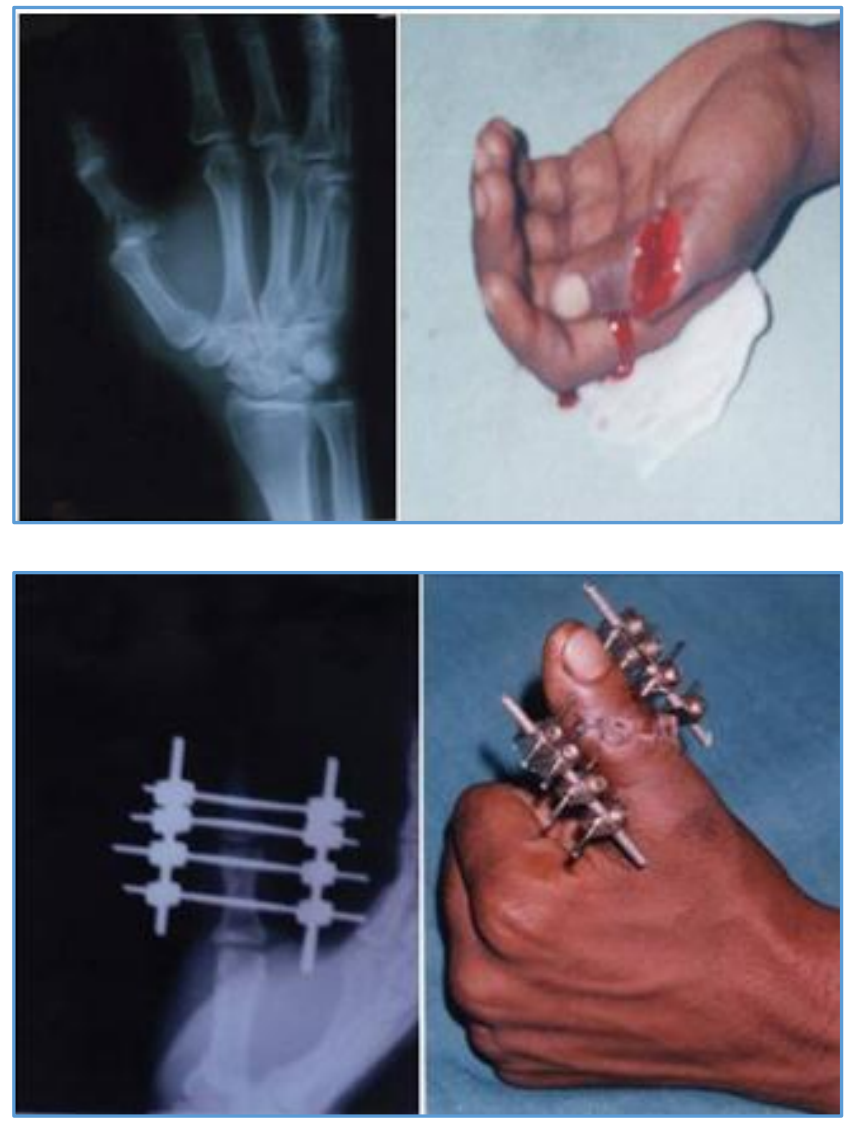

Picture 1: Open Fracture of Proximal Phalanx of Left Thumb, Treated with External Fixator and Primary Suturing

\section{RESULTS}

The mean follow-up was 4.4 years (2.3-8.2). There were no general complications. Ten fractures showed complications during the period of fixation; Pin tract infection-2, Pin loosening-5 and Fracture displacement-3.

The patients with pin tract infection were treated conservatively with antibiotics and dressing. All patients who had loosening of the pins were managed with removal of the device, because the fractures had already become sticky with no instability at the fracture site. Three patients who had fracture displacement were treated with re-reduction and fixation. Both patients who had non-union of fractures underwent open reduction and bone grafting with $\mathrm{K}$-wire fixation. Several patients returned to office and light work before removal of the fixators. Two patients required readmission for ORIF with bone grafting for non-union of the fracture.

\begin{tabular}{|c|c|}
\hline Complication & Number \\
\hline Pin tract infection & 2 \\
\hline Loosening of pin & 5 \\
\hline Loss of reduction & 1 \\
\hline Non-union & 2 \\
\hline Total & 10 \\
\hline \multicolumn{2}{|c|}{ Table 1: Complications Encountered } \\
\hline
\end{tabular}

Functional recovery was evaluated on the basis of the total active range of movements of each injured finger separately using the American Society for Surgery of Hand (ASSH) Criteria. ${ }^{7}$ This adds the active flexion of the metacarpophalangeal, proximal and distal interphalangeal joints and then subtracts the sum of extension deficit at these three joints (Table 2).

\begin{tabular}{|c|c|c|}
\hline Result & Finger & Thumb \\
\hline Excellent & $220-260$ & $119-140$ \\
Good & $180-216$ & $98-118$ \\
Fair & $130-179$ & $70-97$ \\
Poor & $<130$ & $<70$ \\
\hline \multicolumn{2}{|c|}{ Table 2: ASSH Criteria of TAM } \\
\end{tabular}

Table 3 shows the functional results of our cases. None of the fractures had significant axial or rotational deformities. Results were good or excellent in $86.7 \%$ of Metacarpal fractures and in $85.7 \%$ of Phalangeal fractures; 30 out of 39 proximal phalangeal fractures and all the 18 middle phalangeal fractures, 13 out of 15 metacarpal fractures had excellent or good results with overall results of about $85.9 \%$ percentage.

\begin{tabular}{|c|c|c|c|}
\hline Results & Phalangeal & Metacarpal & Percentage \\
\hline Excellent & 30 & 9 & $50.0 \%$ \\
Good & 24 & 4 & $35.9 \%$ \\
Fair & 3 & 0 & $03.8 \%$ \\
Poor & 6 & 2 & $10.3 \%$ \\
\hline \multicolumn{3}{|c|}{ Table 3: Results of the Functional Recovery } \\
\hline
\end{tabular}

Fair or poor results occur in about $14.1 \%$ fractures. Majority of these cases had multiple fractures or were accompanied with severe soft tissue injury; 9 patients with phalangeal and 2 patients with metacarpal fractures had poor or fair results. 

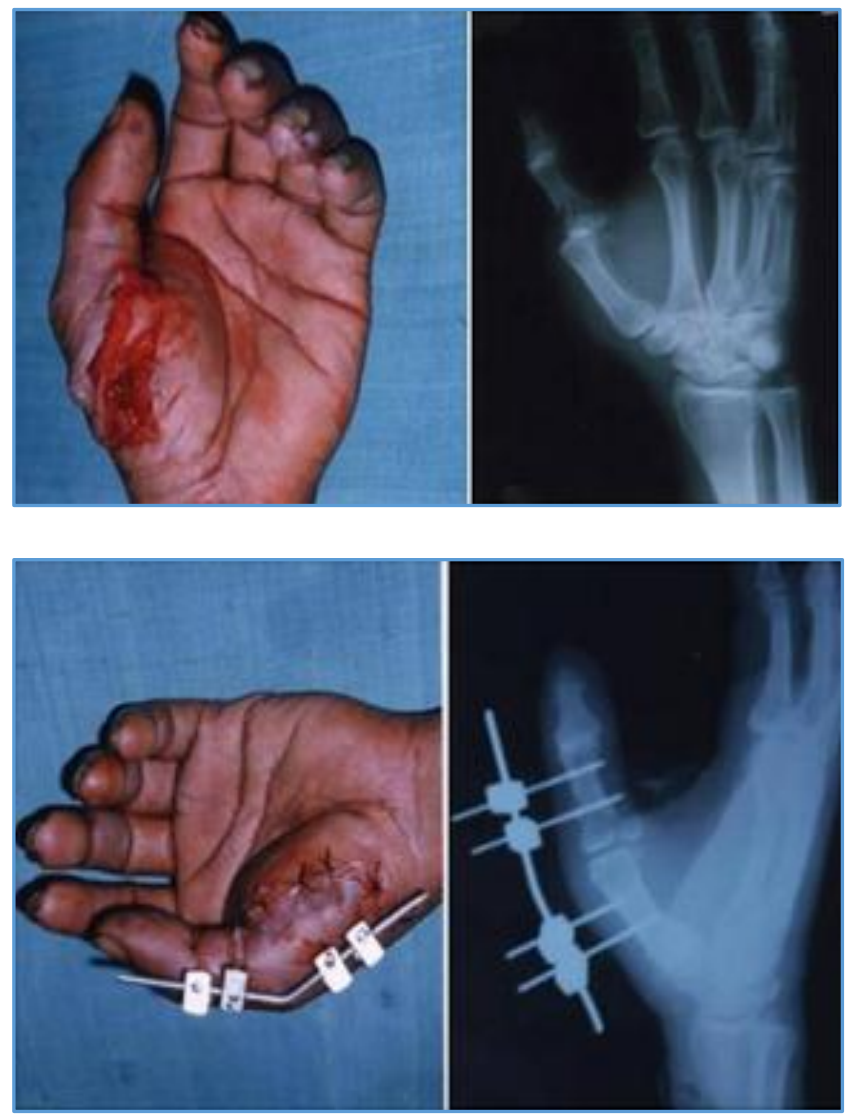

\section{Picture 2: Blast Injury of Left Thumb, Treated with Debridement and External Fixation}

\section{DISCUSSION}

Most of the metacarpal and phalangeal fractures can be treated conservatively, but in a relatively small number of patients such as unstable, intraarticular and open fractures operative treatment is indicated. There has been diversity of operative techniques demonstrated for their treatment.

\section{Kirschner Wire}

It can be used in almost any setting to stabilise open fractures. Wires can be used for intramedullary or transcortical fixation of fractures. Advantages are pin fixation is easy; it requires minimal dissection and is universally available. It can be used to supplement other forms of fixation. Kirschner pins may loosen and may distract the fracture fragments. Furthermore, pin tract infections may develop secondary to skin irritation or loosening and pin protrusion may make therapy and splintage awkward.

\section{Intramedullary Fixation}

Intramedullary fixation is applicable for transverse and short oblique fracture. The method is easy to perform and allows early active motion. There are no exposed pins and secondary removal is unnecessary. Disadvantages include rotational instability, pin migration and occasional fracture distraction.

\section{Plates and Screws}

Plates and screws provide rigid fixation of fracture. It allows early mobilisation of the hand. The application of complex hardware often requires wider exposure and manipulation. In addition, proper instrumentation and a complete assortment of screws size and plate configurations must be available. It may require removal of the implant with the risk of refracture and moreover the implants are bulky.

\section{External Fixation ${ }^{\mathbf{8}, 9,10}$}

External skeletal fixation devices are also now available and have been improved to the point where they are more adaptable and useful for the stabilisation of hand fractures. ${ }^{11}$ Examples include highly comminuted fractures with or without bone loss and open fractures associated with injury to the soft tissue structures.

The advantage of external fixation is that fracture fragments are not stripped of periosteal blood supply and further devascularised. External fixation does not cause osteopenia seen with more rigid systems, such as plating.12,13,14 There is adequate stability to permit early mobilisation. External fixators are adjustable and a second reduction can be carried out if the initial reduction is unstable or has secondarily shifted. Finally, external fixation permits ready access to the wound for debridement as well as for reconstructive surgery.

Complications in our study were minimal and did not affect the outcome in our series because all the fractures were clinically united. The fracture location was an important determinant of final outcome in our study. Our study confirms the findings of previous studies that metacarpal fractures had better functional recovery in comparison to phalangeal fractures. In the phalanges, middle and distal phalanges had better functional recovery than proximal phalanx. The fracture configuration did not affect the functional outcome of our study. The damage caused by the initial injury highly correlate with the final outcome. ${ }^{15}$

Results were good or excellent in about $85.9 \%$. Poor or fair result occurred in about $14.1 \%$ of fractures. Majority of these cases had multiple fractures or were accompanied with severe laceration of tendons. A detailed study about the type of fracture, mode of injury and associated soft tissue injury has been done. The results were analysed overall and in relation to fracture configuration, type of fracture and bone involvement. The cases were regularly followed up at regular interval and analysed based on total active movement by Duncan et al criteria.

Fracture location was an important determinant of outcome in our study. Excellent or good results occurred in about $86.67 \%$ of metacarpal fractures and $85.71 \%$ phalangeal fractures with average of $85.9 \%$. Phalangeal fractures had better functional results. Middle and distal phalangeal fractures had better results than proximal phalangeal factures. ${ }^{16}$

Poor or fair results occurred in about $14.1 \%$ of fracture. In one case, there were multiple fractures in the same hand: intraarticular involvement and severe laceration of tendon. The poorest results were in proximal phalangeal fractures, especially if these were combined with tendon injuries at the same level. ${ }^{17}$

In the study, the damage caused by the initial wound correlated highly with the final outcome. Less severely wounded open hand fractures (Swanson type) had a significantly higher proportion of good and excellent results than those with more severe wounds (type II). Strickland et al and James found a correlation between intraarticular fractures and unfavourable outcomes. In our study, out of five intraarticular fractures four showed good results. 
James and Chow et al found a correlation between extensor and flexor tendon injuries and poor outcomes in open fracture. Our study also indicates that this is particularly true with severe laceration of extensor tendons.

We did not find that age or systemic disease had a statistically significant influence on final functional outcome; this is at variance with other well-documented and wellanalysed series.

Although, the numbers of serious complications were small, we found as did McLain a correlation between wound infection and wound severity. Wound severity seems to be correlated with contamination, associated soft tissues injury and delay in treatment.

External fixation provides adequate basis for the bone healing, because it provides bone stability and early mobilisation. ${ }^{18}$ But the functional result depends mainly on the type of fracture and associated soft tissue injuries.

\section{CONCLUSIONS}

In our prospective study, there were 78 hand fractures which are open and associated with soft tissue injuries treated by external fixation.

The functional recovery was analysed using Duncan et al criteria of total active movement. Majority of the cases had better functional recovery. Poor results occurred in those who had multiple fractures in the same digit or hand, severe comminution, intraarticular fracture and accompanied by severe soft tissue injuries.

Complications occurred in very few cases. Most of the complications were not serious and permanent sequelae were infrequent. Open fracture of hand with soft tissue injuries treated with external fixation had good functional results.

External fixation is an adequate alternative treatment for open metacarpal and phalangeal fractures, which are accompanied by soft tissue injuries.

Concluding, external fixation is adequate alternate treatment for open unstable phalangeal and metacarpal fractures, which are accompanied with severe soft tissue injuries. Our experience confirmed that external fixation is a versatile, reliable and safe technique with a broader application in the treatment of such complicated fractures.

\section{REFERENCES}

1. Ashmead D, Rothkopf DM, Walton RL, et al. Treatment of hand injuries by external fixation. J Hand Surg Am 1992;17(5):954-64.
2. Buchler $U$. The small AO external fixator in hand surgery. Injury 1994;25(Suppl 4):S-D55-D63.

3. Freeland AE. External fixation for skeletal stabilization of severe open fractures of the hand. Clin Orthop Relat Res 1987;(214):93-100.

4. Swanson TV, Szabo RM, Anderson DD. Open hand fractures: prognosis and classification. J Hand Surg Am 1991;16(1):101-7.

5. Parsons SW, Fitzgerald JA, Shearer JR. External fixation of unstable metacarpal and phalangeal fractures. J Hand Surg Br 1992;17(2):151-5.

6. Cziffer E. Static fixation of finger fractures. Hand Clinc 1993;9(4):639-50.

7. Duncan RW, Freeland AE, Jabaley ME, et al. Open hand fractures: an analysis of the recovery of active motion and of complications. J Hand Surg Am 1993;18(3):387-94.

8. Eyres KS, Kreibich N, Allen TR. Stabilization of multiple metacarpal fractures. A new use for the charnley toefusion clamp. J Hand Surg 1993:18(2):192-4.

9. Hastings $H$. Open fractures and those with soft tissue damage: treatment by external fixation. In: Barton NJ. edr. Fractures of the hand and wrist. New York: Churchill Livingstone1988:145-72.

10. Hochberq J, Arderghy M. Stabilization of hand phalangeal fractures by external fixator. W V Med J 1994;90(2):54-7.

11. Joshi BB. Percutaneous internal fixation of fractures of the proximal phalanges. Hand 1976;8(1):86-92.

12. Pritsch M, Engel J, Farin I. Manipulation and external fixation of metacarpal fractures. J Bone Joint Surg 1981;63(8):1289-91.

13. Schuind F, Cooney WP, Burny F, et al. Small external fixation devices for the hand wrist. Clin Orthop Relat Res 1993;(293):77-82.

14. Schuind F, Donkerwolcke M, Burny F. External mini fixation for treatment of closed fractures of the metacarpal bones. J Orthop Trauma 1991;5(2):146-52.

15. Seitz WH, Gomez W, Putnam MD, et al. Management of severe hand trauma with a mini external fixator. Orthopedics 1987;10(4):601-10.

16. Shehadi SI. External fixation of metacarpal and phalangeal fractures. J Hand Surg Am 1991;16(3):544-50.

17. Smith RS, Alonso J, Horowitz M. External fixation of open comminuted fractures of the proximal phalanx. Orthop Rev 1987;16(12):937-41.

18. Watson JA. A simple external fixator for metacarpal and phalangeal fractures. Injury 1993;24(9):635-6. 